\title{
Effects of Bifurcations on Aft-Fan Engine Nacelle Noise
}

\author{
Douglas M. Nark, F. Farassat, D. Stuart Pope; and V. Vatsa ${ }^{\S}$ \\ NASA Langley Research Center, Hampton, Virginia
}

\begin{abstract}
Aft-fan engine nacelle noise is a significant factor in the increasingly important issue of aircraft community noise. The ability to predict such noise within complex duct geometries is a valuable tool in studying possible noise attenuation methods. A recent example of code development for such predictions is the ducted fan noise propagation and radiation code CDUCT-LaRC. This work focuses on predicting the effects of geometry changes (i.e. bifurcations, pylons) on aft fan noise propagation. Beginning with simplified geometries, calculations show that bifurcations lead to scattering of acoustic energy into higher order modes. In addition, when circumferential mode number and the number of bifurcations are properly commensurate, bifurcations increase the relative importance of the plane wave mode near the exhaust plane of the bypass duct. This is particularly evident when the bypass duct surfaces include acoustic treatment. Calculations involving more complex geometries further illustrate that bifurcations and pylons clearly affect modal content, in both propagation and radiation calculations. Additionally, results show that consideration of acoustic radiation results may provide further insight into acoustic treatment effectiveness for situations in which modal decomposition may not be straightforward. The ability of CDUCT-LaRC to handle complex (non-axisymmetric) multi-block geometries, as well as axially and circumferentially segmented liners, allows investigation into the effects of geometric elements (bifurcations, pylons).
\end{abstract}

\section{Introduction}

Aft-fan engine nacelle noise is a significant factor in the increasingly important issue of aircraft community noise. The ability to predict such noise within complex duct geometries is a

\footnotetext{
${ }^{*}$ Research Scientist, Structural Acoustics Branch, Aerodynamics, Aerothermodynamics, and Acoustics Competency, AIAA Member

${ }^{\dagger}$ Senior Research Scientist, Aeroacoustics Branch, Aerodynamics, Aerothermodynamics, and Acoustics Competency, AIAA Associate Fellow

$\ddagger$ Analytical Services and Materials

${ }^{\S}$ Senior Research Scientist, Computational Modeling and Simulation Branch, Aerodynamics, Aerothermodynamics, and Acoustics Competency
} 
valuable tool in studying possible noise attenuation methods. A recent example of code development for such predictions is the ducted fan noise propagation and radiation code CDUCT-LaRC. ${ }^{1}$ Previous aft-fan propagation calculations using CDUCT-LaRC have indicated that studying the effects of bifurcation geometry and placement, as well as the use of segmented liners, may prove to be a fruitful area of research for increased attenuation. Therefore, utilizing the capability within CDUCT-LaRC to handle complex geometries, this work focuses on predicting the effects of geometry changes (i.e. bifurcations, pylons) on aft fan noise propagation. Noise propagation through bypass duct geometries of varying complexity is studied. Enhancements to the post-processing capabilities of CDUCT-LaRC provide some details into the predicted effects of the bifurcations. In addition to attenuation prediction, modal decomposition functionality is also available. Therefore, some quantification of bifurcation effects may be obtained through attenuation prediction and decomposition into hard-wall duct modes.

Initially, results for straight, axi-symmetric ducts are presented to illustrate the use of the modal decomposition functionality. Various bifurcation effects are illustrated through the use of the simplified geometries with and without treatment. Subsequently, a bypass duct geometry similar to that of a business jet, containing one internal bifurcation, is studied. Results for hard and soft-wall bifurcations are presented. This is followed by the discussion of results for a larger commercial jet engine geometry which incorporates an internal bifurcation, as well as a pylon that extends beyond the exhaust plane of the bypass duct. Finally, concluding remarks are offered, along with further areas of application and development.

\section{General Bifurcation Effects and Modal Decomposition}

Prior to studying more complex geometries, it is useful to attempt to identify some general bifurcation effects using simplified cases. In this light, preliminary calculations were performed using a straight annular duct geometry with two different internal configurations. The first configuration, configuration 1, contains a single infinitely thin bifurcation in the middle third of the duct. The second, configuration 2, incorporates a second lower bifurcation located 180 degrees circumferentially from the upper bifurcation in the same axial position. The radial dimensions are taken to be similar to those of a business jet engine with inner and outer radii of $0.28 \mathrm{~m}$ (11 in) and $0.41 \mathrm{~m}$ (16 in), respectively. The ducts have an overall length of $1.09 \mathrm{~m}$ (43 in) and a uniform background flow was prescribed $(M=0.4)$ inside the duct since the flow is taken to be inviscid and the bifurcations are infinitely thin. Another reason for the starting with a straight annular geometry, and hence uniform flow, is to facilitate the decomposition of the propagation solutions into hard wall annular duct modes. Straight-forward decomposition with non-uniform mean flows may be ill-defined and may provide non-physical modal amplitudes. The uniform background flow afforded by the straight geometry with infinitely thin bifurcations avoids this issue. 


\section{A. Configuration 1}

One point of interest is the change in modal content brought about by the bifurcations. Therefore, propagation calculations were performed within configuration 1 with the acoustic source specified as the $(5,1)$ and $(10,1)$ hardwall annular duct modes at the duct inlet with a source frequency of $3000 \mathrm{~Hz}$. Initially, all surfaces were specified as hardwall and the resulting contours of the real part of the acoustic potential are shown in figure 1.

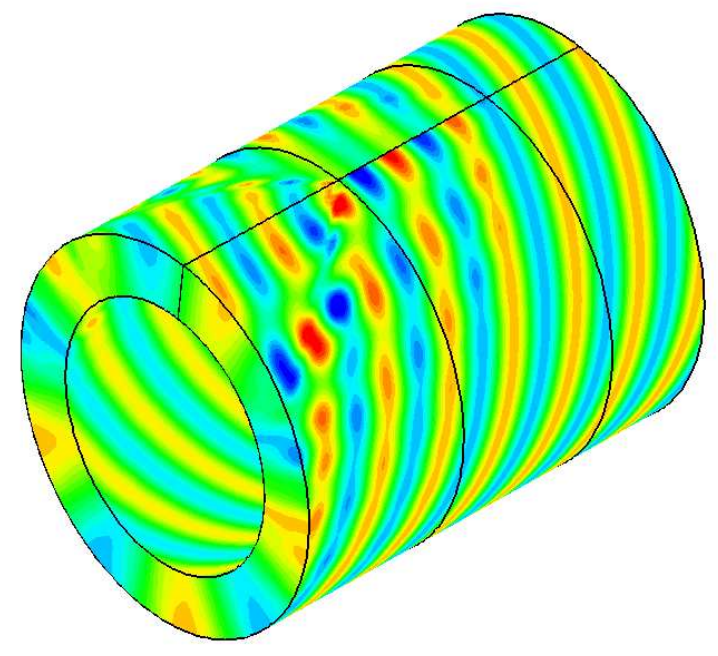

(a) $(5,1)$ Mode

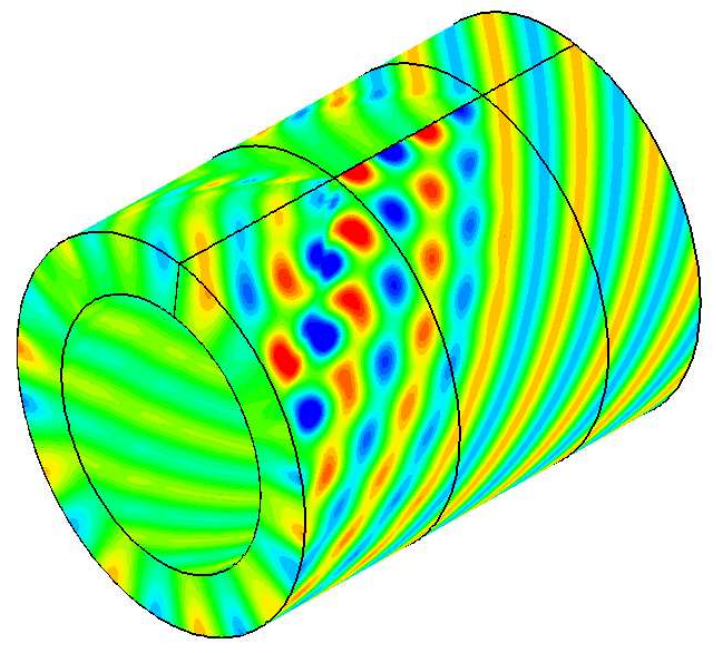

(b) $(10,1)$ Mode

Figure 1. Real Part of Acoustic Potential - Configuration 1 [ $f=3000 \mathrm{~Hz}]$

In this figure, the fluid flow and acoustic propagation are from right to left (i.e. the exhaust plane is in the foreground). While the typical modal pattern is present, the effects of the bifurcation are visible as the contours appear to show evidence of standing waves within the bifurcation region. In an effort to verify this, modal decomposition may be employed at the exhaust plane to gain some idea of the modal character resulting from the presence of the bifurcation. Decomposing the propagation result into hardwall annular duct modes leads to figure 2 .

The circumferential and radial mode numbers are on the $x$ and $y$ axes, respectively. The $z$ axis represents the amplitude of the acoustic potential normalized by its maximum value. It should be noted that the radial mode numbering convention used in this work considers the first radial mode to be one (i.e. $n=1)$. The plot contains the value $n=0$ simply as a place holder for the plane wave case $(m=0, n=0)$. Therefore, in all modal plots, the only non-zero amplitude at $n=0$ will appear for the plane wave. Returning to figure 2, this plot shows that some acoustic energy is transferred to circumferential modes rotating in the opposite sense to the input modes. In addition, 


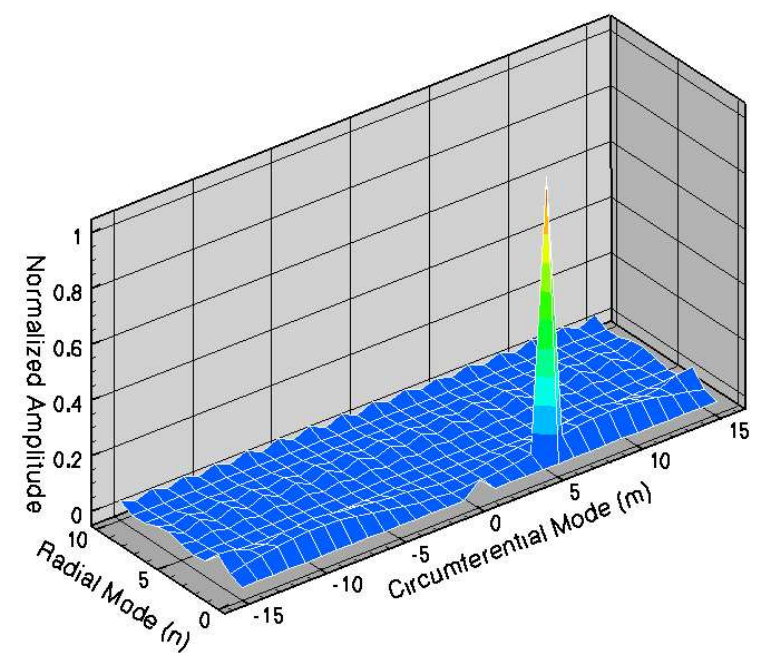

(a) $(5,1)$ Mode

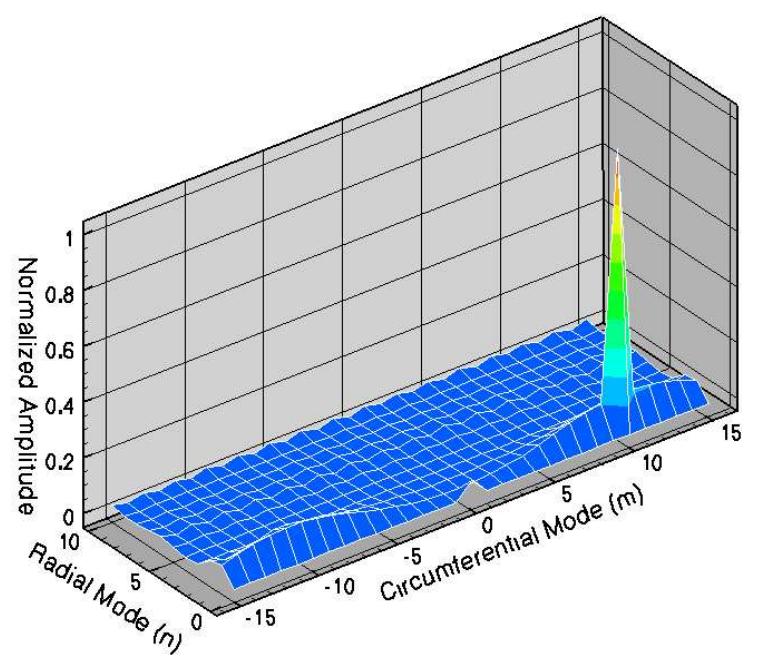

(b) $(10,1)$ Mode

Figure 2. Modal Decomposition at the Exhaust Plane - Configuration 1 (Hardwall)

some acoustic energy is scattered to higher order circumferential and radial modes. Finally, a small amount of acoustic energy in the plane wave mode may be noticed.

This analysis may be taken a step further by considering the fact that a bypass duct typically includes treatment on the inner and outer walls. Also, as discussed by Dougherty, ${ }^{2}$ treatment on the bifurcation surfaces may also be quite effective. Therefore, to gain a more realistic idea of the relative amplitudes of the modes at the exhaust plane, acoustic treatment was applied to the surfaces of configuration 1. As a baseline example, consider the inner and outer walls to incorporate a 1 inch deep single layer liner (reactance following $\cot (k L)$ relation) having a resistance of $1.0 \rho c$. Since the bifurcations are infinitely thin, consider the bifurcation treatment to have a resistance of $1.5 \rho c$ and zero reactance. Propagation calculations were again performed for the $(5,1)$ and $(10,1)$ hardwall duct modes with a source frequency of $3000 \mathrm{~Hz}$. Attenuation calculations show that the treatment is quite effective, with $20-25 \mathrm{~dB}$ attenuation in the two cases. This led to exhaust plane modal amplitudes that were less than $10 \%$ of the hardwall cases. Contours of the real part of acoustic potential are not included as the results are similar to those in figure 1 with the amplitude decreasing in the propagation direction. Modal decomposition was also performed at the exhaust plane of the bypass duct and the results are presented in figure 3.

In order to show relative levels more clearly, the amplitudes have again been normalized with respect to maximum amplitude in the exhaust plane for each case. It can be seen from these figures that the effectiveness of the liner in attenuating the higher order modes has brought the levels down to the point at which the plane wave mode becomes more relevant. This seems feasible in that the 


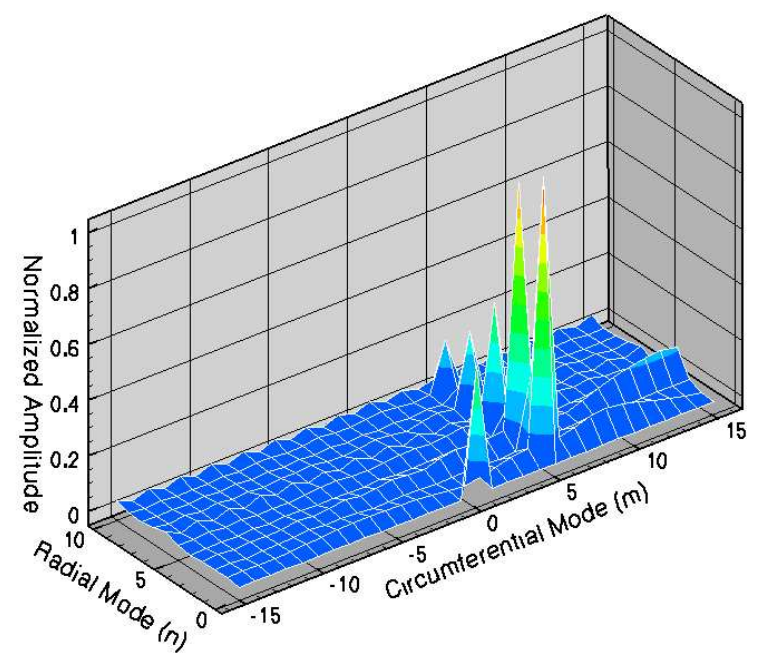

(a) $(5,1)$ Mode

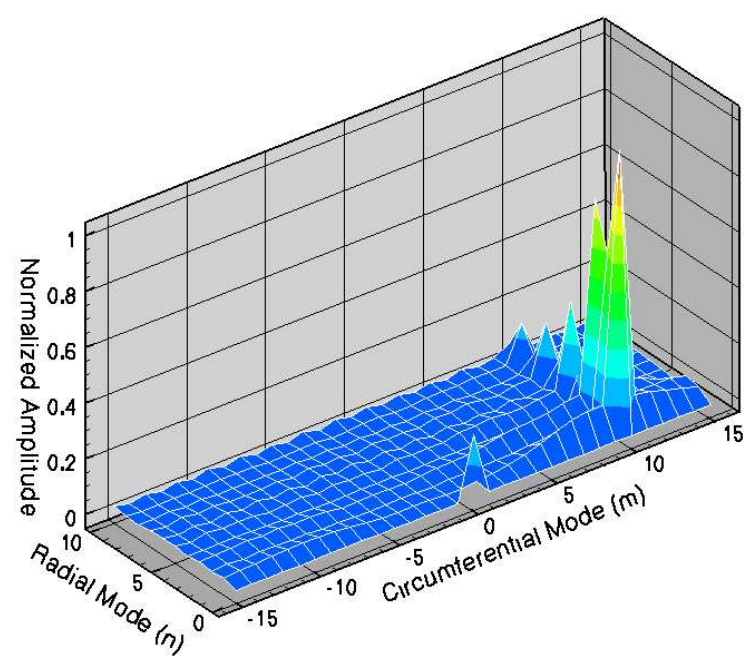

(b) $(10,1)$ Mode

Figure 3. Modal Decomposition at the Exhaust Plane - Configuration 1 (Treated)

plane wave mode is 'well cut-on', thus providing the liner less opportunity for attenuation.

The radiation calculation module within CDUCT-LaRC may provide some further insight into the changes in the acoustic solution brought about by the bifurcation. The acoustic radiation module is based on a solution to the Ffowcs-Williams Hawkings equation with a penetrable data surface. Currently, the exhaust plane of the bypass duct is used as the source plane. Radiation calculations were performed on a hemisphere of radius $1.91 \mathrm{~m}$ (75 in) centered on the duct axis in the exhaust plane. Figure 4 shows the normalized radiated SPL on this hemisphere for the hardwall and treated cases. In this figure, and radiation results to follow, the radiated SPL was normalized with the respect to the maximum value obtained for the individual hardwall cases. Therefore, the hardwall results will show maximum normalized values of 1 and the treated results generally have maximum normalized values less than 1 . In general, the effects of the bifurcation are clearly visible in that the radiation pattern is considerably different than the symmetric 'bullseye' pattern obtained from an axisymmetric annular duct. ${ }^{1}$

\section{B. Configuration 2}

Noting the results for configuration 1, similar propagation, decomposition, and acoustic radiation calculations were performed for configuration 2 . The modal decomposition results with hardwall internal surfaces for the $(5,1)$ and $(10,1)$ modes with a source frequency of $3000 \mathrm{~Hz}$ are shown in figure 5 . The results are similar to those shown for configuration 1 in that some acoustic energy 


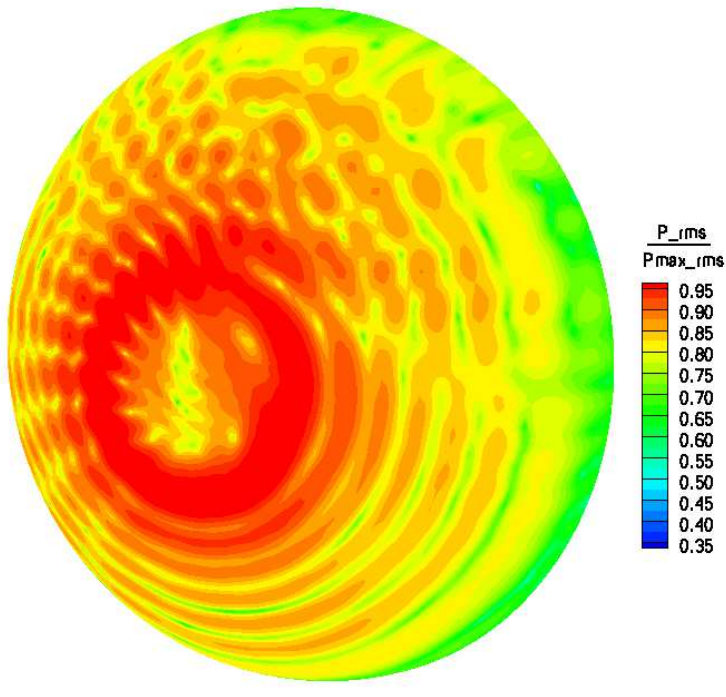

(a) Hardwall

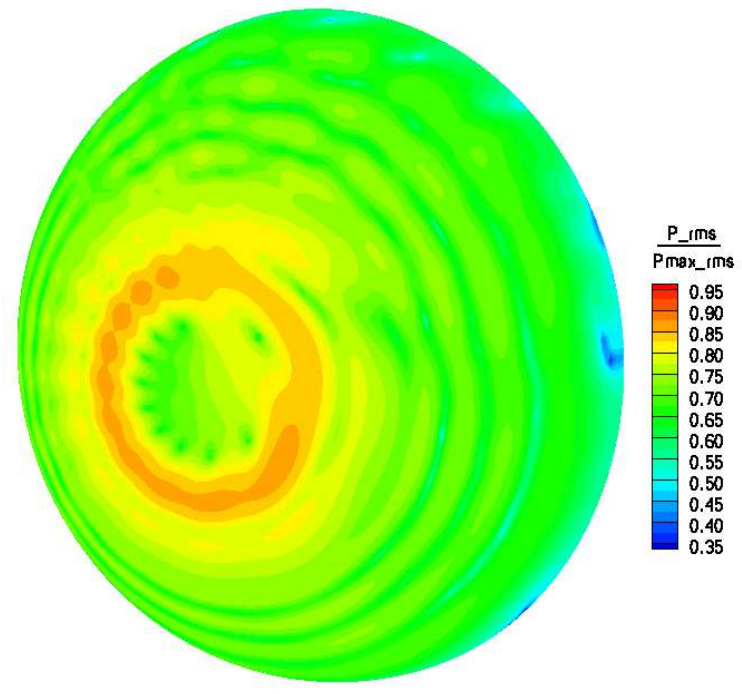

(b) Treated

Figure 4. Normalized Radiation Patterns - Configuration 1 [(10,1) Mode]

appears to scatter into the higher order modes. Additionally, there is acoustic energy present in circumferential modes rotating in the opposite sense to the input modes, as well as the plane wave mode.

Modal decomposition for the treated case are shown in figure 6. As was the case for configuration 1 , the treatment was very effective as 20-25 dB attenuation was achieved. For this configuration, it appears that $(5,1)$ mode did not lead to a situation in which the plane wave mode became as relatively important as in the $(10,1)$ mode and configuration 1 results.

The analysis presented by Tyler and Sofrin ${ }^{3}$ may provide an explanation for this behavior. Taking the source circumferential mode to represent the number of blades, $b$, and the number of bifurcations to be the equivalent number of vanes, $v$. It can be seen that interaction between the $(5,1)$ mode and the two bifurcations to produce a zero order circumferential mode does not appear. However, with the $(10,1)$ mode, interaction to produce a zero order circumferential mode is possible, as $b-5 v=10-5(2)=0$. This approach may also explain the results for configuration 1 discussed earlier, as the number of bifurcations $(v=1)$ provides many opportunities for interaction.

Acoustic radiation calculations were also performed for comparison with configuration 1 results. The normalized radiation patterns in figure 7 again show considerable difference from the symmetric pattern obtained from a straight axisymmetric duct. In addition, the radiation patterns for configuration 2 show a different asymmetry than those of configuration 1 . In this case, there are two areas, near the top and bottom, in which the symmetry of the pattern has been disrupted. This is in contrast to figure 4 , in which the patterns show one large area of asymmetry. 


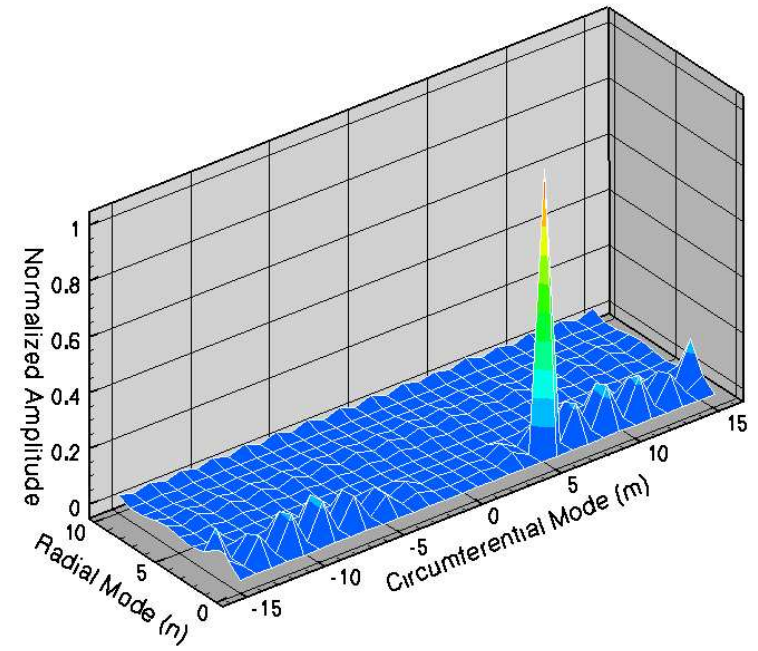

(a) $(5,1)$ Mode

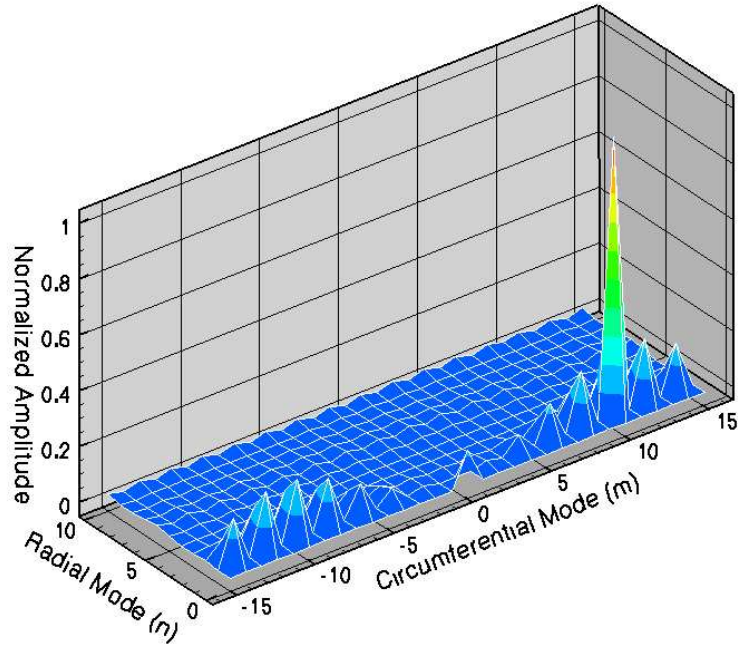

(b) $(10,1)$ Mode

Figure 5. Modal Decomposition at the Exhaust Plane - Configuration 2 (Hardwall)

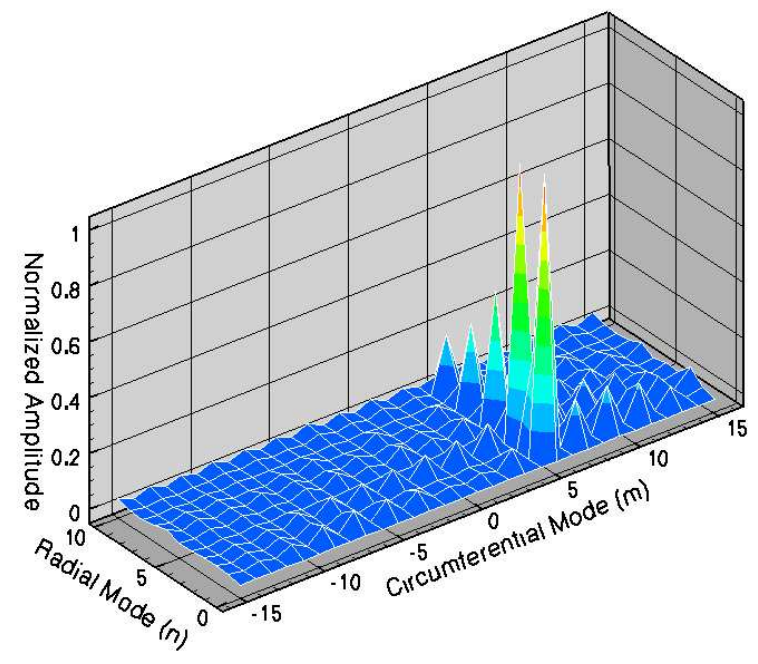

(a) $(5,1)$ Mode

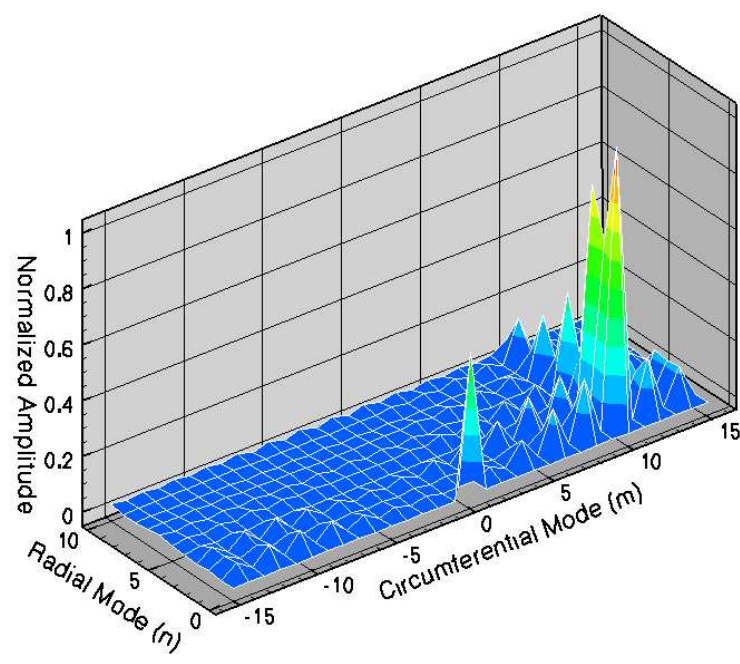

(b) $(10,1)$ Mode

Figure 6. Modal Decomposition at the Exhaust Plane - Configuration 2 (Treated) 


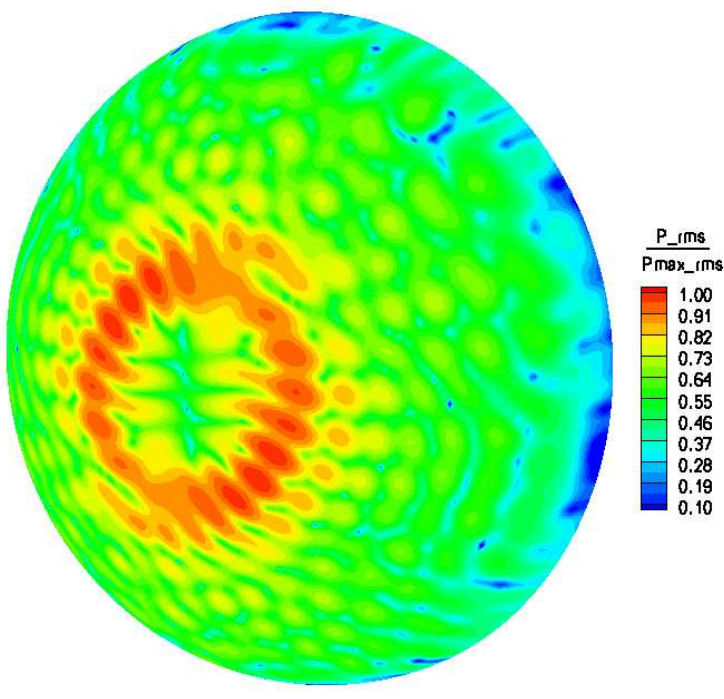

(a) Hardwall

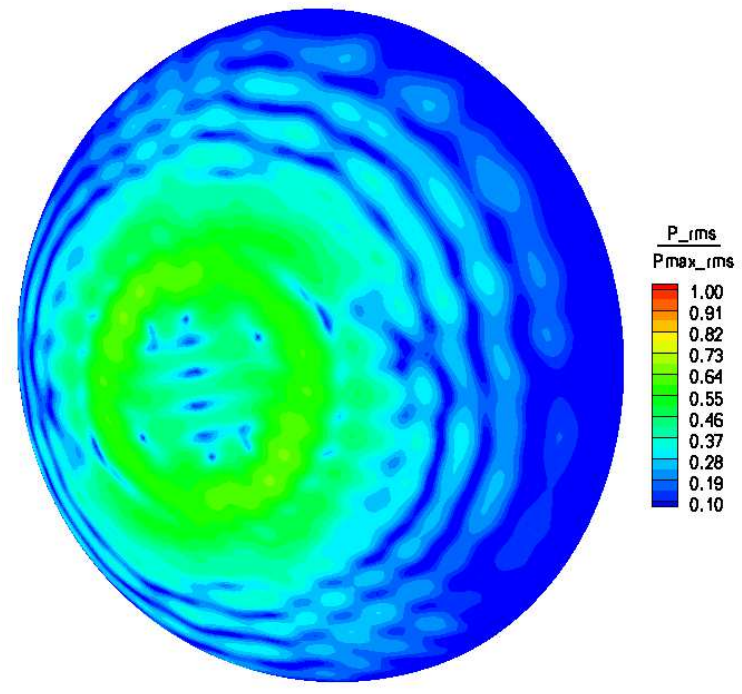

(b) Treated

Figure 7. Normalized Radiation Patterns - Configuration 2 [(10,1) Mode]

Results up to this point have focused on the $(5,1)$ and $(10,1)$ modes specified independently. To extend this analysis further, propagation calculations may be performed with multiple modes specified as the acoustic source. Figure 8 presents results for the treated case with the $(5,1),(5,2)$, $(10,1)$ and $(10,2)$ modes specified with equal amplitudes. The figure also includes a treated case with the $(-5,1)$ and $(10,1)$ modes specified with equal amplitudes.

Acoustic radiation calculations were performed and again the effects of the bifurcations are clearly visible. Normalized radiation patterns for the $(5,1),(5,2),(10,1)$ and $(10,2)$ mode acoustic source are presented in figure 9 . The $(-5,1)$ and $(10,1)$ acoustic source results are also shown in figure 10. Once again, there is a modification of the directivity pattern associated with the interaction between the bifurcations and the modal content.

\section{Internal Bifurcation}

Having identified various general bifurcation effects within the straight annular ducts of configurations 1 and 2, similar analysis may be turned to more realistic geometries. As a step in this progression, consider the generic business jet engine geometry shown in figure 11.

The bifurcation was modeled as a NACA 0012 airfoil and the duct has inner and outer radii of $0.305 \mathrm{~m}$ (12 in) and $0.391 \mathrm{~m}$ (15.4 in), respectively. In figure 11, the cross-section of the bifurcation is visible to identify the location of the internal bifurcation. Contours of the real part 


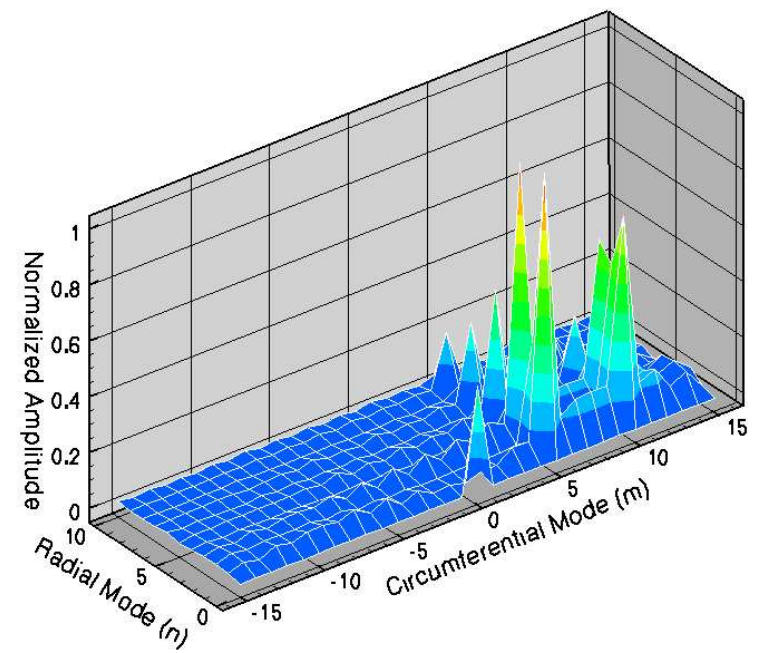

(a) $(5,1),(5,2),(10,1)$, and $(10,2)$ Modes

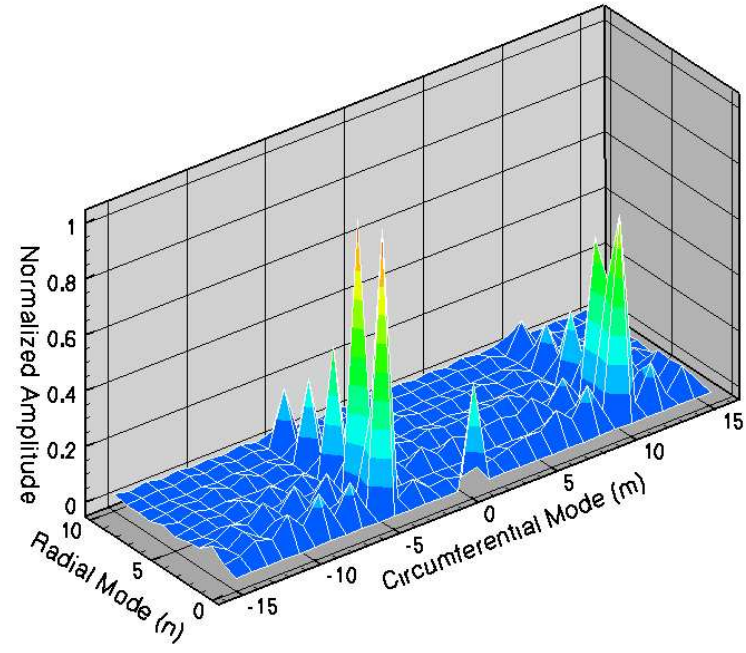

(b) $(-5,1)$ and $(10,1)$ Modes

Figure 8. Modal Decomposition at the Exhaust Plane - Configuration 2 (Treated)

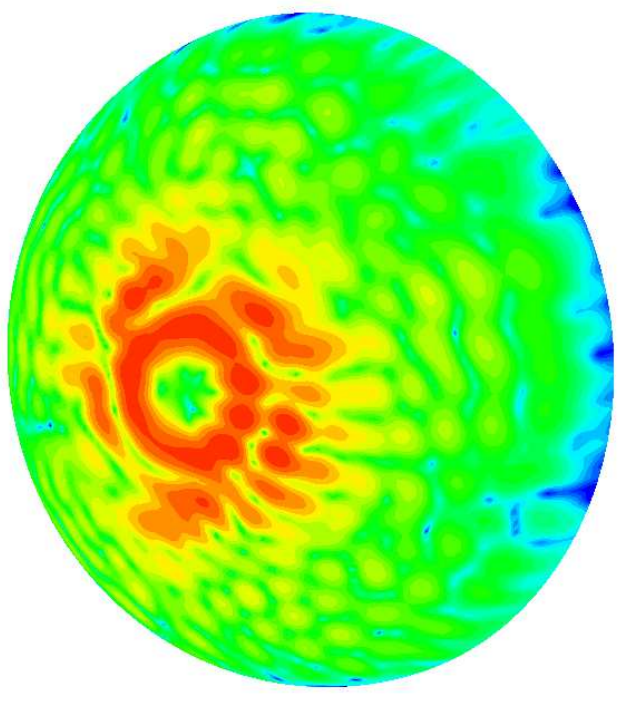

(a) Hardwall

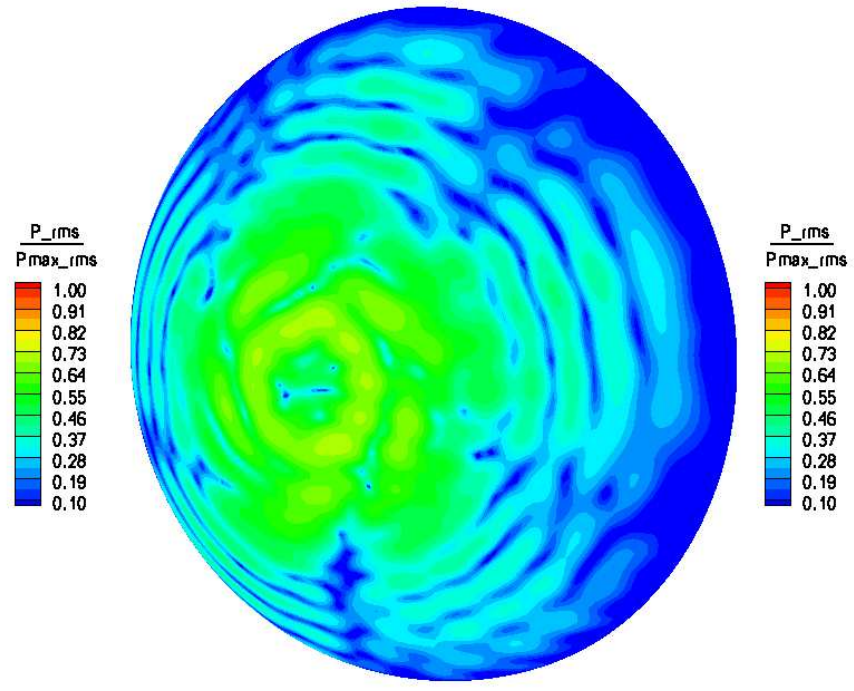

(b) Treated

Figure 9. Normalized Radiation Patterns - Configuration $2[(5,1),(5,2),(10,1)$ and $(10,2)$ Modes $]$ 


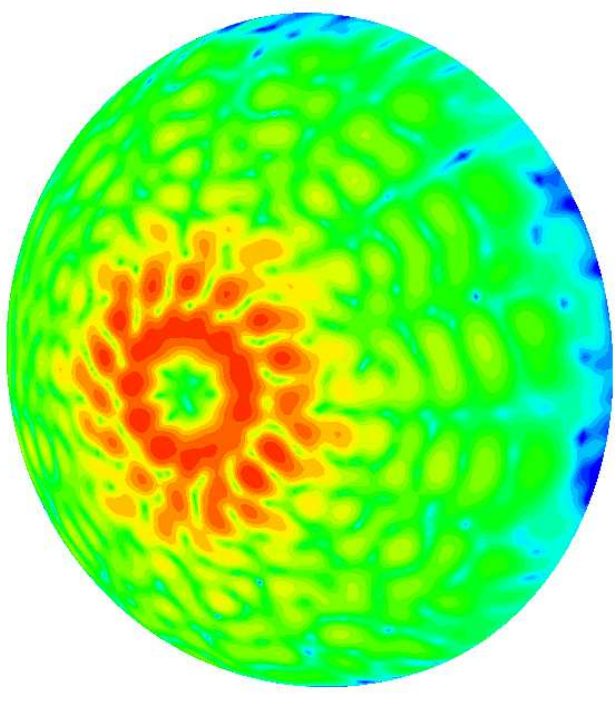

(a) Hardwall

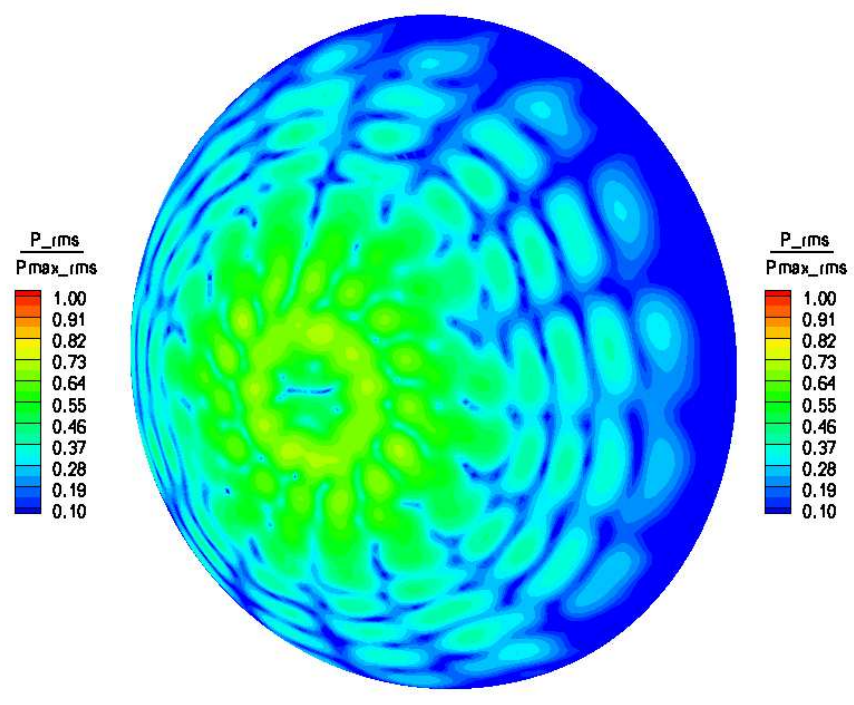

(b) Treated

Figure 10. Normalized Radiation Patterns - Configuration 2 [(-5,1) and (10,1) Modes]

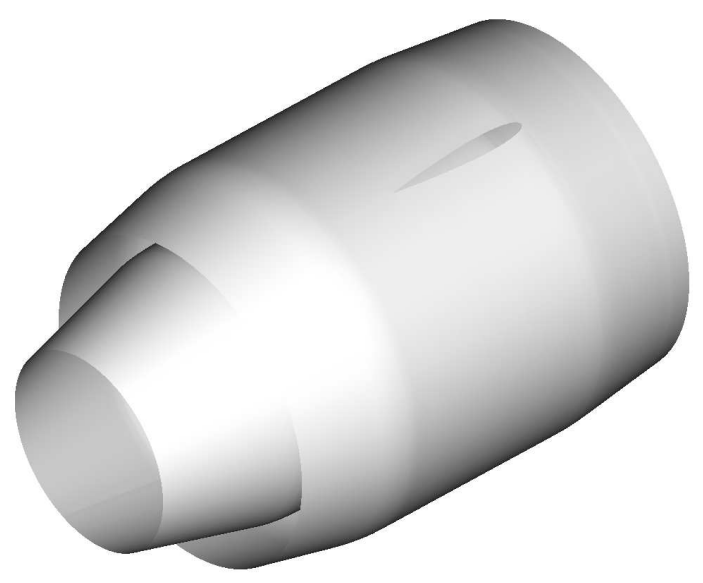

Figure 11. Generic Business Jet Engine Geometry 
of acoustic potential are presented for a $(5,1),(5,2),(10,1)$ and $(10,2)$ mode acoustic source in figure 12. The mean flow module of CDUCT-LaRC was used to obtain the background flow with an inlet Mach number of 0.4. While the typical modal pattern is present in portions of the duct, bifurcation effects are visible as standing waves near the bifurcation region. The finite thickness of the bifurcation and variable cross-sectional area of the duct lead to non-uniform mean flow in the bypass-duct. As mentioned previously, this may cause difficulties for the application of straight forward decomposition into hardwall annular duct modes. In light of this, modal decomposition was not performed for this case. However, acoustic radiation results were obtained on a hemisphere of radius approximately 5 duct diameters, centered on the duct axis in the exhaust plane. As seen in figure 13, there is again asymmetry in directivity. However, this case also illustrates that radiated pressure may provide insight into liner effectiveness in cases involving complex geometries and non-uniform mean flow.

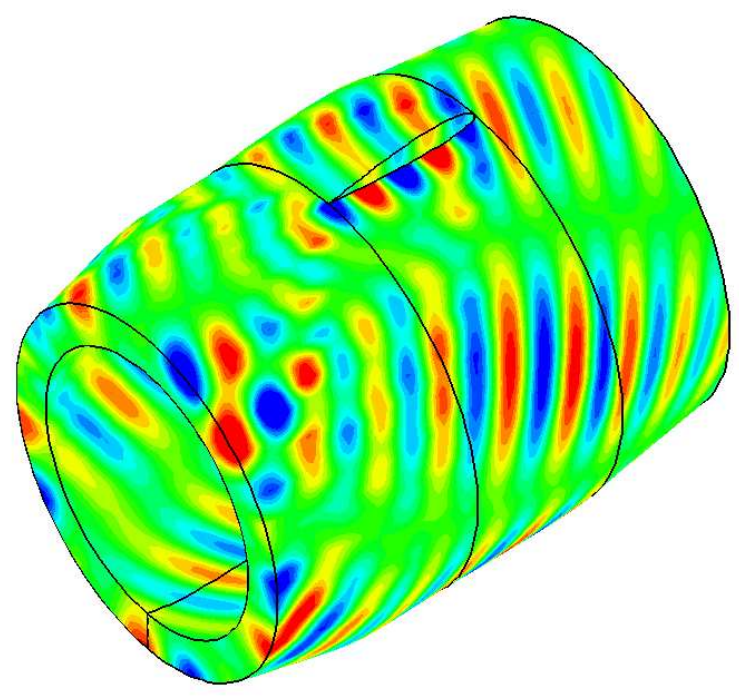

Figure 12. Real Part of Acoustic Potential - Business Jet (Hardwall) $[(5,1),(5,2),(10,1)$, and $(10,2)$ Modes, $f=$ $3000 \mathrm{~Hz}]$

\section{Internal Bifurcation with Pylon}

As a final example in the progression toward more complex geometries, the Jet Engine Simulator (JES) of the Jet Noise Laboratory at NASA Langley Research Center, shown in figure 14, was studied.

The pylon is clearly visible extending from the bypass duct, as well as the internal lower bifurcation. Figure 15 shows contours of the real part of acoustic potential for a $(5,1),(5,2),(10,1)$ and $(10,2)$ mode acoustic source. The modal pattern is clearly affected, as acoustic scattering is evident 


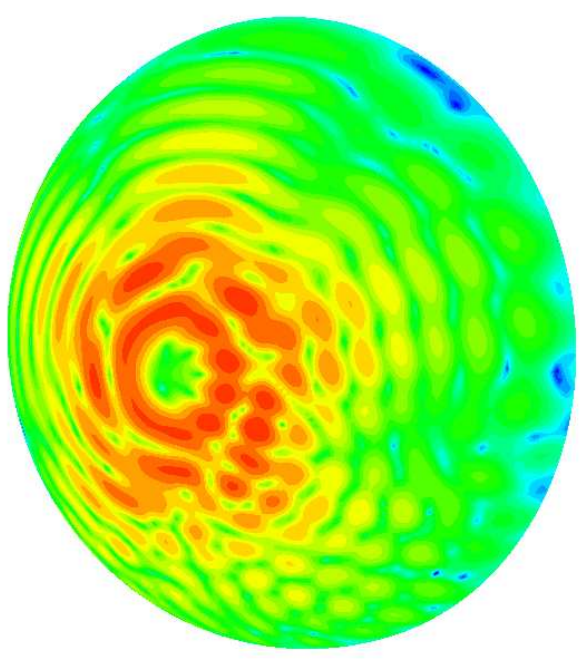

(a) Hardwall

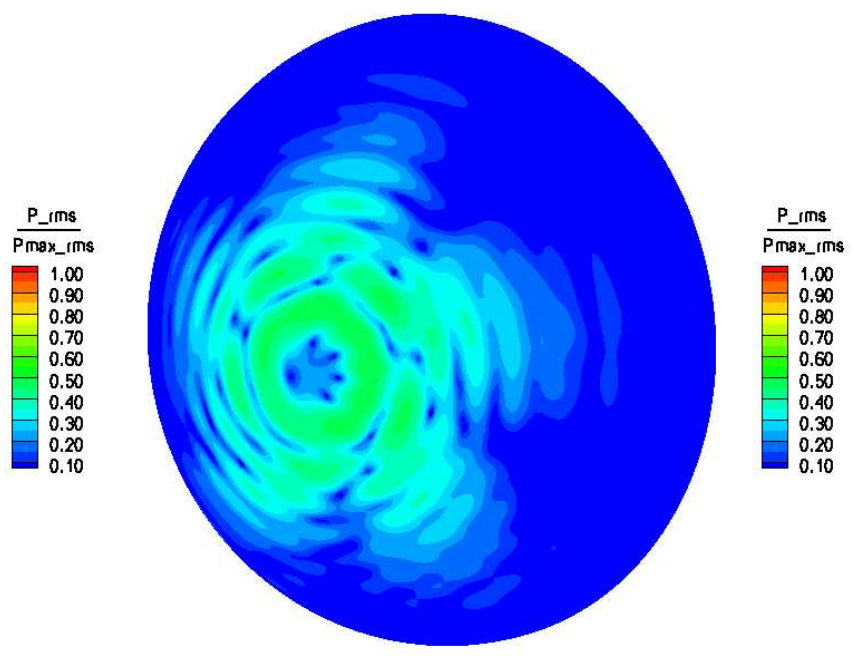

(b) Treated

Figure 13. Normalized Radiation Patterns - Business Jet $[(5,1),(5,2),(10,1)$ and $(10,2)$ Modes]

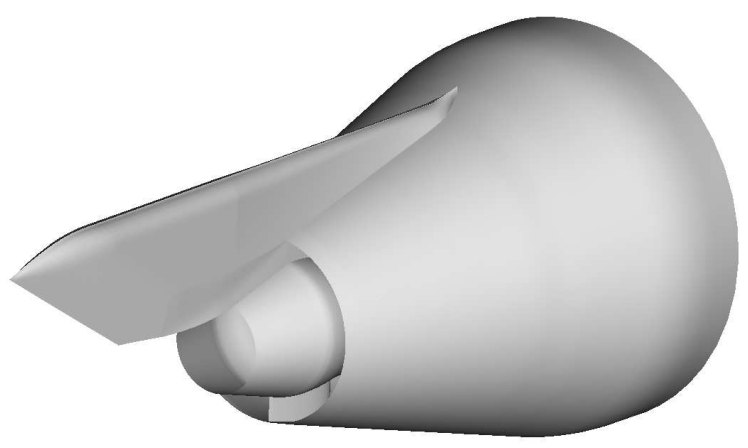

Figure 14. Jet Engine Simulator (JES) Geometry 
in the vicinity of the pylon and bifurcation. As seen in figure 16, associated radiation calculations were also performed on a hemisphere of radius approximately 3 duct diameters, centered on the duct axis in the exhaust plane. Note that these results incorporate the exhaust plane of the bypass duct as the source surface. One possible approach to extending the propagation calculation to the core cowl, and hence including more of the external pylon and core cowl effects on acoustic radiation, is discussed in reference. ${ }^{4}$ However, this example illustrates the type of complex geometries that one may attempt to study.

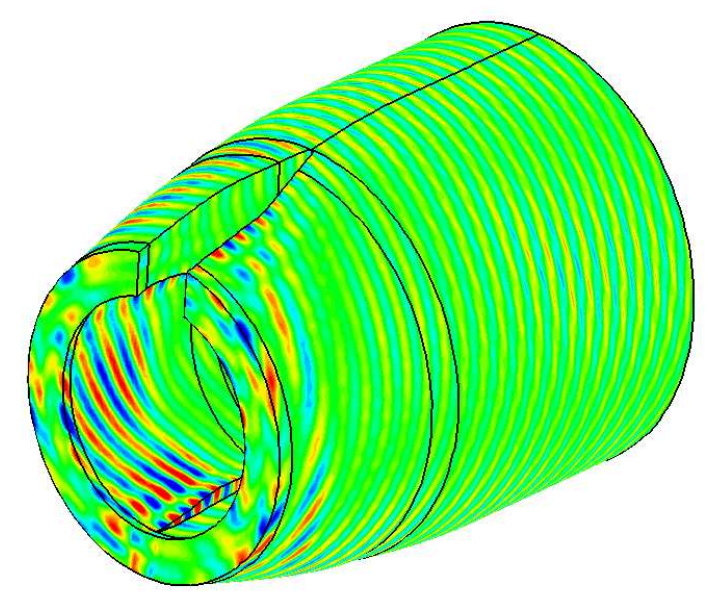

Figure 15. Real Part of Acoustic Potential - JES (Hardwall) $[(5,1),(5,2),(10,1)$, and $(10,2)$ Modes, $f=1600 \mathrm{~Hz}]$

\section{Concluding Remarks}

This work has illustrated the use of CDUCT-LaRC to study the effects of bifurcations and pylons on acoustic propagation and radiation for geometries of varying complexity. In initial cases, scattering of acoustic energy into higher modes was evident. In addition, the relative importance of the plane wave mode was increased for treated cases with both one and two bifurcations. Calculations involving more complex geometries further illustrated that bifurcations and pylons clearly affect the modal content, in both the propagation and radiation results. Additionally, these cases showed that consideration of acoustic radiation results may provide some further insight into treatment effectiveness for situations in which modal decomposition may not be straightforward. The ability of CDUCT-LaRC to handle complex (non-axisymmetric) multi-block geometries, as well as axially and circumferentially segmented liners, provides an opportunity to study the effects of geometric elements (i.e. bifurcations, splitters) on fan noise. In addition, further validation looks to the design and/or quantification of possible methods of achieving increased overall attenuation in realistic geometries. 


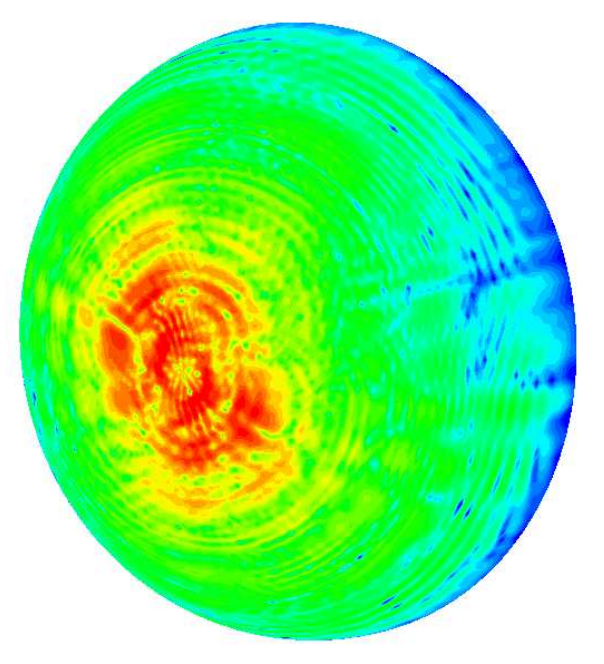

(a) Hardwall
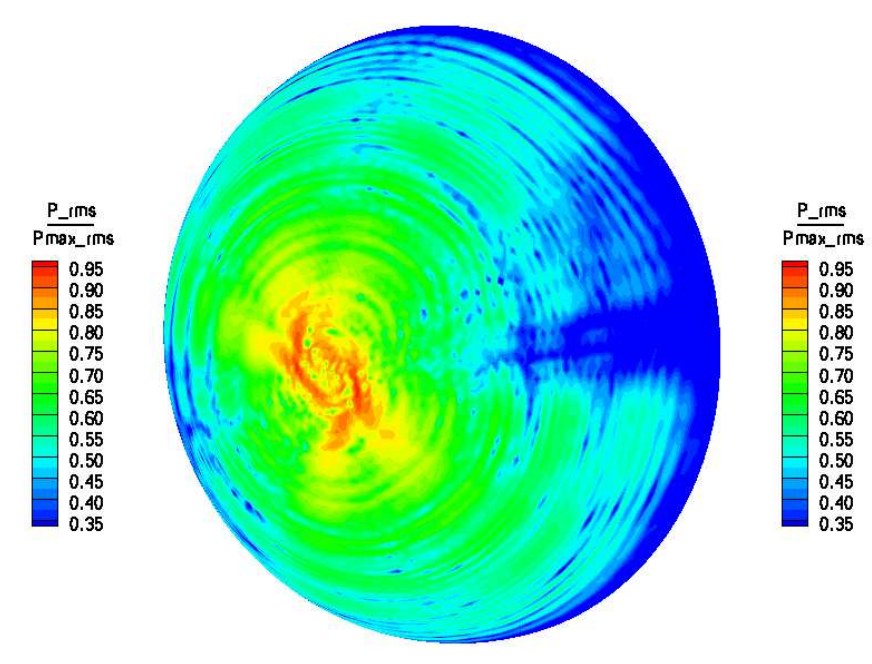

(b) Treated

Figure 16. Normalized Radiation Patterns - JES $[(5,1),(5,2),(10,1)$ and $(10,2)$ Modes $]$

\section{Acknowledgments}

The authors would like to thank Russ Thomas of the Aeroacoustics Branch at NASA Langley and Mike Wiese of the NASA Langley Geometry Laboratory for assistance with the Jet Engine Simulator geometry.

\section{References}

${ }^{1}$ Nark, D. M., Farassat, F., Pope, D. S., and Vatsa, V., "The Development of the Ducted Fan Noise Propagation and Radiation Code CDUCT-LaRC," AIAA Paper 2003-1652, 2003.

${ }^{2}$ Dougherty, R. P., "A Parabolic Approximation for Flow Effects on Sound Propagation in Nonuniform, Softwall, Ducts," AIAA Paper 99-1822, 1999. 332.

${ }^{3}$ Tyler, J. M. and Sofrin, T. G., “Axial Flow Compressor Noise Studies,” SAE Transactions, Vol. 70, 1962, pp. 309-

${ }^{4}$ Nark, D. M., Farassat, F., Pope, D. S., and Vatsa, V., “A Model for Shear Layer Effects on Engine Noise Radiation," AIAA Paper 2004-2992, 2004. 